\title{
CrimRxiv
}

\section{"I'll Choose My Own Way": Delinquent Girls and Boys in Search of Gender Hegemony}

\section{Armelle Weil}

Published on: Feb 11, 2022

DOI: 10.21428/cb6ab371.33393670

License: Creative Commons Attribution 4.0 International License (CC-BY 4.0). 
